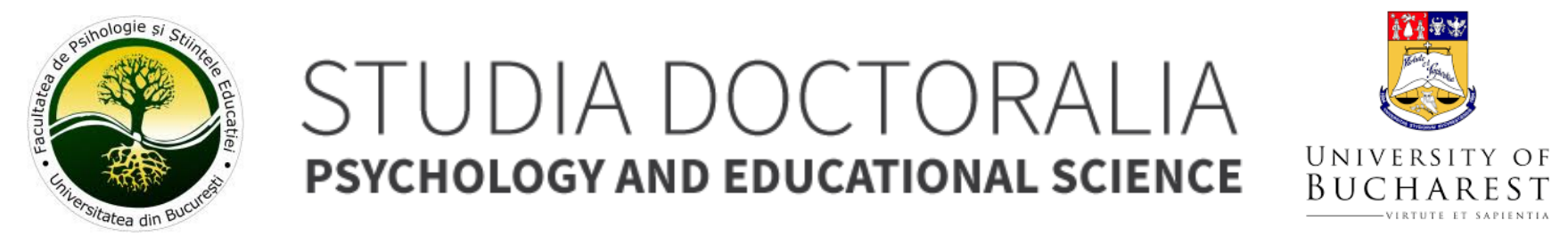

\title{
EDITORIAL
}

\section{Modeling the Covid-19 epidemic in Romania}

\author{
Mihnea Cosmin Muraru
}

The Covid-19 epidemic in Romania reached the number of 18,283 total confirmed cases as of 25 May 2020. Out of these, 11,630 had recovered, 1,193 had died (Romanian Ministry of Health, 2020) and 5,460 were still active, with 213 new cases. In order to contain the epidemic, the authorities adopted increasingly stricter regulations, such as closing schools and universities, imposing the state of emergency, canceling flights to/from high-risk regions, and increasing the number of daily administered tests. On 15 May 2020, the restrictions were relaxed, and Romania entered the state of alert, while the authorities continued to monitor the situation in case undesirable consequences would occur.

The strict measures were justified by two considerations. First, the virus is highly contagious, as indicated by early estimates of the control reproduction number $\left(R_{0}\right)$, between 2 and 3.5 (Read et al., 2020; Zhao et al., 2020). This figure denotes the average number of secondary infections produced by a typical infected person in a completely susceptible population, during the course of the disease. If $R_{0}$ is greater than 1 , the epidemic grows exponentially; if it is equal to 1 , the epidemic is maintained and may become endemic (constantly infecting a small proportion of the population); finally, for the epidemic to subside, it must be less than 1 . Second, there seems to be an important transmission potential of asymptomatic individuals ( $\mathrm{He}$ et al., 2020), and there might be a large proportion of asymptomatic or mildly symptomatic cases, estimated between $18 \%$ and $80 \%$ (for example, Mizumoto et al., 2020; Nishiura et al., 2020). Taken together, these facts appear to contribute significantly to the spread of the disease.
In order to better understand the evolution of the epidemic and to adopt adequate measures for containing it, mathematical and statistical models can be an important tool. We have developed a simple statistical model able to predict the evolution of the total number of confirmed cases by day (Fig. 1). Since the initial regime of the epidemic is exponential, the data is usually plotted on a semilogarithmic scale (right-hand side of Fig. 1), where the trend appears approximately linear. Thus, we were able to apply straightforward linear regression on the transformed data. The slope of the regression line tells us the rate of exponential growth, i.e. the percent increase in the number of cases. The model was trained using the data reported until 28 March. For the interval 26 February - 16 March, the average daily increase in the number of cases was found to be $27 \%$, i.e. the number of new cases on a given day was on average $27 \%$ of the existing number of cases on the previous day. On 17 March, lockdown started, and the model needed to take into account the new dynamic. Thus, simple linear regression was no longer adequate, and we employed multiadaptive regression splines (MARS), which is able to fit multiple regression lines on different intervals in the data. Using this technique, we found that the new growth rate was brought down to $18 \%$, suggesting that the enforced measures had a positive impact. Until 28 March, the predictive ability of the model was very $\operatorname{good}\left(R^{2}=.99\right)$. All the predictions are available on our website: https://appliedpsychology.ro/covid19.html.

However, after $28 \mathrm{March}$, the data indicated that the epidemic is gradually leaving the exponential regime, and entering the linear one, where the number of new daily cases becomes approximately constant. As can be seen in Fig. 1, the long-term predictions of the 
model start to diverge from the reported data, after this point. We could have employed more complex statistical models for time series prediction, such as ARIMA models (for example, Benvenuto et al., 2020), or even deep neural network models (for example, Punn, Sonbhadra \& Agarwal, 2020), which are able to detect more complex and subtle interactions in the data. However, these models are fundamentally limited because they do not capture the epidemiologic essence of the phenomena. Also, there is no straightforward way to simulate the effects of potential measures, of different nature and strength.

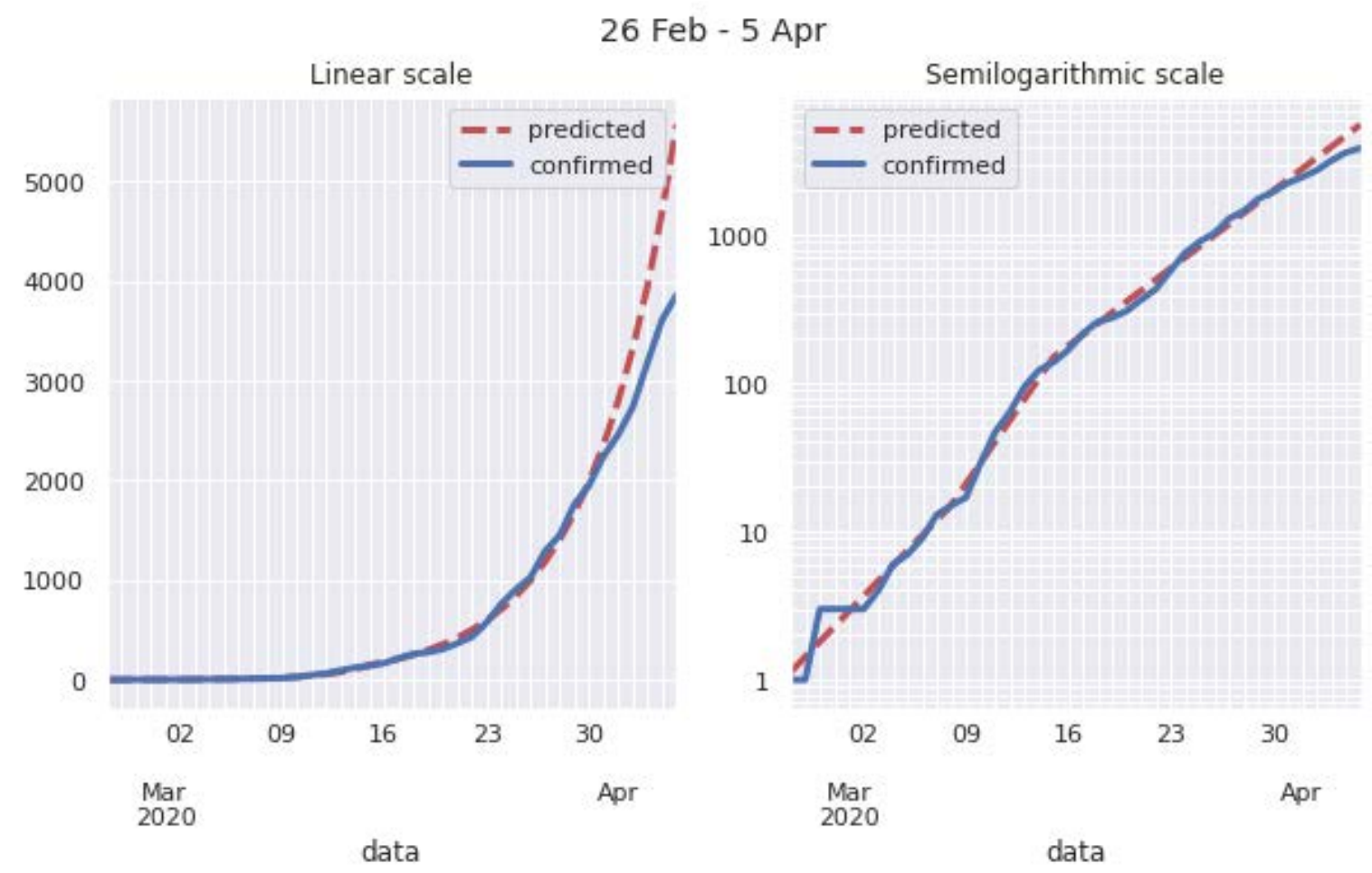

Figure 1. Predicted and confirmed total number of cases between 26 February and 5 April 2020

Consequently, we have started developing an epidemiological model, aimed at fitting the reported data onto the theoretical evolution of the epidemic. This is a mean-field type of model, similar to the classic susceptible-infected-recovered model (SIR; Kermack \& McKendrick, 1927). The population is split into several compartments, such as susceptible to disease, asymptomatic infected, symptomatic infected, diagnosed, recovered, deceased etc., and detailed interactions are modeled through different rates of transition between compartments. These rates will be estimated by both fitting the model to reported data and by imposing epidemiological and clinical constraints onto the model parameters, since the reported data are not sufficient to narrow down the search space (see, for example, Giordano et al., 2020). The model will be able to estimate the number of true cases (beyond confirmed ones), the control reproduction number $\left(R_{c}\right)$ after different measures were adopted by the authorities, the longterm ( 1 year) evolution of the epidemic according to actual and simulated policies of different nature and strength (such as social distancing and testing), and also counterfactual dynamics (what would have happened, hadn't the lockdown been enforced). 


\section{REFERENCES}

Benvenuto, D., Giovanetti, M., Vassallo, L., Angeletti, S., \& Ciccozzi, M. (2020). Application of the ARIMA model on the COVID-2019 epidemic dataset. Data in brief, 105340.

Giordano, G., Blanchini, F., Bruno, R., Colaneri, P., Di Filippo, A., Di Matteo, A., \& Colaneri, M. (2020). Modelling the COVID-19 epidemic and implementation of population-wide interventions in Italy. Nature Medicine, 1-6.

He, X., Lau, E. H., Wu, P., Deng, X., Wang, J., Hao, X., ... \& Mo, X. (2020). Temporal dynamics in viral shedding and transmissibility of COVID-19. Nature medicine, 1-4.

Kermack, W. O., \& McKendrick, A. G. (1927). A contribution to the mathematical theory of epidemics. Proceedings of the royal society of london. Series $A$, Containing papers of a mathematical and physical character, 115(772), 700-721.

Mizumoto, K., Kagaya, K., Zarebski, A., \& Chowell, G. (2020). Estimating the asymptomatic proportion of coronavirus disease 2019 (COVID19) cases on board the Diamond Princess cruise ship, Yokohama, Japan, 2020. Eurosurveillance, 25(10), 2000180.
Nishiura, H., Kobayashi, T., Miyama, T., Suzuki, A., Jung, S., Hayashi, K., ... \& Linton, N. M. (2020). Estimation of the asymptomatic ratio of novel coronavirus infections (COVID-19). medRxiv. Retrieved from https://www.medrxiv.org/ content/10.1101/2020.02.03.20020248v2

Punn, N. S., Sonbhadra, S. K., \& Agarwal, S. (2020). COVID-19 Epidemic Analysis using Machine Learning and Deep Learning Algorithms. medRxiv.

Read, J. M., Bridgen, J. R., Cummings, D. A., Ho, A., \& Jewell, C. P. (2020). Novel coronavirus 2019-nCoV: early estimation of epidemiological parameters and epidemic predictions. MedRxiv. Retrieved from https://www.medrxiv.org/content/ 10.1101/2020.01.23.20018549v2.

Romanian Ministry of Health (2020, May 25). News. Retrieved from http://www.ms.ro/ category/stiri/.

Zhao, S., Lin, Q., Ran, J., Musa, S. S., Yang, G., Wang, W., ... \& Wang, M. H. (2020). Preliminary estimation of the basic reproduction number of novel coronavirus (2019-nCoV) in China, from 2019 to 2020: A data-driven analysis in the early phase of the outbreak. International journal of infectious diseases, 92, 214-217. 